\title{
Theory for Transitions Between Exponential and Stationary Phases: Universal Laws for Lag Time
}

\begin{abstract}
Yusuke Himeoka and Kunihiko Kaneko*
Department of Basic Science, University of Tokyo, Komaba, Meguro-ku, Tokyo 153-8902, Japan (Received 11 November 2016; revised manuscript received 25 February 2017; published 27 June 2017)

The quantitative characterization of bacterial growth has attracted substantial attention since Monod's pioneering study. Theoretical and experimental works have uncovered several laws for describing the exponential growth phase, in which the number of cells grows exponentially. However, microorganism growth also exhibits lag, stationary, and death phases under starvation conditions, in which cell growth is highly suppressed, for which quantitative laws or theories are markedly underdeveloped. In fact, the models commonly adopted for the exponential phase that consist of autocatalytic chemical components, including ribosomes, can only show exponential growth or decay in a population; thus, phases that halt growth are not realized. Here, we propose a simple, coarse-grained cell model that includes an extra class of macromolecular components in addition to the autocatalytic active components that facilitate cellular growth. These extra components form a complex with the active components to inhibit the catalytic process. Depending on the nutrient condition, the model exhibits typical transitions among the lag, exponential, stationary, and death phases. Furthermore, the lag time needed for growth recovery after starvation follows the square root of the starvation time and is inversely related to the maximal growth rate. This is in agreement with experimental observations, in which the length of time of cell starvation is memorized in the slow accumulation of molecules. Moreover, the lag time distributed among cells is skewed with a long time tail. If the starvation time is longer, an exponential tail appears, which is also consistent with experimental data. Our theory further predicts a strong dependence of lag time on the speed of substrate depletion, which can be tested experimentally. The present model and theoretical analysis provide universal growth laws beyond the exponential phase, offering insight into how cells halt growth without entering the death phase.
\end{abstract}

DOI: 10.1103/PhysRevX.7.021049

Subject Areas: Biological Physics, Nonlinear Dynamics

\section{INTRODUCTION}

Quantitative characterization of a cellular state, in terms of the cellular growth rate, concentration of external resources, and abundances of specific components, has long been one of the major topics in cell biology, ever since the pioneering study by Monod [1]. Such studies have been developed mainly by focusing on the microbial, exponentially growing phase, in which the number of cells grows exponentially (this phase is often termed the log phase in cell biology, but considering the focus on exponential growth, here adopt the term "exponential phase" throughout). This work has uncovered somewhat universal growth laws, including Pirt's equation for yield and growth [2] and the relationship between the fraction of ribosomal abundance and growth rate (experimentally demonstrated by Schaechter et al. [3], and theoretically rationalized by Scott et al. [4]), among

*kaneko@complex.c.u-tokyo.ac.jp

Published by the American Physical Society under the terms of the Creative Commons Attribution 4.0 International license. Further distribution of this work must maintain attribution to the author(s) and the published article's title, journal citation, and DOI. others [5-8], in which the constraint to maintain steady growth leads to general relationships [9-11].

In spite of the importance of the discovery of these universal laws, cells under poor conditions exhibit different growth phases in which such relationships are violated. Indeed, in addition to the death phase, cells undergo a stationary phase under conditions of resource limitation, in which growth is drastically suppressed. Once cells enter the stationary phase, a certain time span is generally required to recover growth after resources are supplied, which is known as the lag time. There have been extensive studies conducted to characterize the stationary phase, including the length of lag time for resurrection and the tolerance time for starvation or antibiotics [12-14], and specific possible mechanisms for phase transitions have been proposed [15-17]. Furthermore, recent experiments have uncovered the quantitative relationships of lag time and its cell-to-cell variances $[18,19]$. For example, the lag time was shown to depend on the length of time the cells are starved. This implies that the stationary phase is not actually completely stationary but that some slow changes still progress during the starvation time, in which cells "memorize" the starvation time. Hence, a theory to explain such slow dynamics is needed that can also characterize 
the phase changes and help to establish corresponding quantitative laws.

The existence of these phases and lag time is ubiquitous in bacteria (as well as most microorganisms). Hence, we aim to develop a general model that is as simple as possible, without resorting to specific detailed mechanisms, but we can nonetheless capture the changes among the lag, exponential, stationary, and death phases. We first describe a simple model for a growing cell, which consists of an autocatalytic process driven by active chemical components such as ribosomes. However, this type of model with autocatalytic growth from substrates and their derivatives, which is adopted for the exponential phase, is not sufficient to represent all phases, as the autocatalytic process either grows exponentially or decays toward death, and thus does not account for a halting state with suppressed growth corresponding to the stationary phase. Therefore, to go one step further beyond the simplest model, we then consider the addition of an extra class of components that do not contribute to catalytic growth. Still, even the inclusion of this extra class of components cannot fully account for the transition to the stationary phase. Therefore, we further considered the interaction between the two classes of components. Here, we propose a model that includes the formation of a complex between these two types of components, which inhibits the autocatalytic process by the active components. We show that the model exhibits the transition to the stationary phase with growth suppression. By analyzing the dynamics of the model, we then uncover the quantitative characteristics of each of these phases in line with experimental observations, including the bacterial growth curve, quantitative relationships of lag time with starvation time and the maximal growth rate, and the exponentially tailed distribution of lag time. The proposed model also allows us to derive several experimentally testable predictions, including the dependence of lag time on the speed of the starvation process.

\section{A. Model}

Since molecules that contribute to autocatalytic processes are necessary for the replication of cells, models for growing cells generally consist of at least substrates $(S)$ and active components (denoted as "component A" hereafter) that catalyze their own synthesis as well as that of other components. For example, in the models developed by Scott et al. [4] and Maitra et al. [20], component A corresponds to ribosomes, whereas several models involving catalytic proteins have also been proposed [10,21-24]. This class of models provides a good description of the exponential growth of a cell under the condition of sufficient substrate availability; however, once the degradation rate of component $\mathrm{A}$ exceeds its rate of synthesis under a limited substrate supply, the cell's volume will shrink, leading to cell death. Hence, a cell population either grows exponentially or dies out, and in this cellular state, it is not possible to maintain the population without growth. However, cells often exhibit suppressed growth under substrate-poor conditions, even at a single-cell level $[12,13,18]$, as observed in the stationary phase. Such cells, which neither grow exponentially nor move toward death, cannot be modeled with cell models that only consider autocatalytic processes [4,10,20-24].

Therefore, to model a state with such suppressed growth, it is important to consider additional chemical species, i.e., macromolecules that do not contribute to autocatalytic growth, in addition to the substrates $(S)$ and component $\mathrm{A}(A)$ that are commonly adopted in models of cell growth. Component A represents molecules that catalyze their own growth, such as ribosomes, and can include metabolic enzymes, transporters, and growth-facilitating factors. Component B represents waste products or can other molecules that are produced with the aid of component A but do not facilitate growth. Thus, the next simplest model is given by

$$
\begin{aligned}
& \frac{d S}{d t}=-F_{A}(S) A-F_{B}(S) A+A\left(S_{\mathrm{ext}}-S\right)-\mu S, \\
& \frac{d A}{d t}=F_{A}(S) A-d_{A} A-\mu A, \\
& \frac{d B}{d t}=F_{B}(S) A-d_{B} B-\mu B .
\end{aligned}
$$

Here, $S_{\text {ext }}$ and $S$ indicate the concentrations of the extracellular and intracellular substrates, respectively. The concentration of the intracellular substrate determines the synthesis rate of the active and nonautocatalytic proteins $F_{A}$ and $F_{B}$, respectively. All chemical components are diluted because of the volume growth of a cell.

In addition to dilution, macromolecules (A and B) are spontaneously degraded with slow rates $\left(d_{A}\right.$ and $\left.d_{B}\right)$. In this model, the cell takes up substrates from the external environment from which component $\mathrm{A}$ and the nongrowth-facilitating component $\mathrm{B}$ are synthesized. These syntheses, $S_{\text {ext }} \leftrightarrow S, S \rightarrow A$, and $S \rightarrow B$, as well as the uptake of substrates take place with the aid of catalysis by component A. Then, by assuming that the synthesized components are used for growth in a sufficiently rapid period, the growth rate is set to be proportional to the synthesis rate of component $\mathrm{A}$. Hence, the dilution rate $\mu$ of each component due to cell volume growth is set as $\mu=F_{A} A$.

Now, if the ratio $F_{A} / F_{B}$ does not depend on the substrate concentration $S$, the fraction $A / B$ does not depend on $S$ either, and the model is reduced to the original autocatalytic model; thus, the phase change to suppressed growth is not expected. Then, by introducing the $S$ dependence of $F_{A} / F_{B}$ to reduce the rate of component A with the decrease in the substrate condition, we first tested whether the transition to a suppressed growth state, as in the stationary phase, occurs under a substrate-poor condition, by setting 
$F_{A} / F_{B}$ to decrease in proportion to the change in $S$ (i.e., $\left.[d /(d S)] F_{A} / F_{B}>0\right)$. However, in this case, it is straightforwardly confirmed that there is no transition to a suppressed growth state. In other words, the cells always grow exponentially without any slowing-down process, as the decrease in $S$ simply influences the growth rate $\mu$, while the presence of $B$ does not influence the dynamics of $A$ (see also Appendix A).

Thus, we need to introduce an interaction between component $\mathrm{A}$ and the non-growth-facilitating component B. Although complicated interactions that may involve other components could be considered, the simplest and most basic interaction that can also provide a basis for considering more complex processes would be the formation of a complex between $\mathrm{A}$ and $\mathrm{B}$ given by the reaction $A+B \leftrightarrow C$. This results in the inhibition of the autocatalytic reaction for cell growth, as complex $\mathrm{C}$ does not contribute to the activity for the autocatalytic process. A schematic representation of the present model is shown in Fig. 1(a). Thus, our model is given by

$$
\begin{aligned}
& \frac{d S}{d t}=-F_{A}(S) A-F_{B}(S) A+A\left(S_{\mathrm{ext}}-S\right)-\mu S, \\
& \frac{d A}{d t}=F_{A}(S) A-G(A, B, C)-d_{A} A-\mu A, \\
& \frac{d B}{d t}=F_{B}(S) A-G(A, B, C)-d_{B} B-\mu B, \\
& \frac{d C}{d t}=G(A, B, C)-d_{C} C-\mu C,
\end{aligned}
$$

where $G(A, B, C)$ denotes the reaction of complex formation, given by $k_{p} A B-k_{m} C$. The catalytic activity of component $\mathrm{A}$ is inactivated because of the formation of complex C. Here, the complex has higher stability than that of other proteins $\left(d_{C}\right.$ is smaller than $d_{A}$ and $\left.d_{B}\right)$ [25].

From Eq. (2), by summing up $\dot{A}$ and $\dot{C}$, we obtain $\dot{A}+\dot{C}=F_{A}(S) A(1-(A+C))$ if $d_{A}$ and $d_{C}$ are zero (or negligible). This means that once the cell reaches any steady state, the relationship $A+C=1$ is satisfied as long as $A$ and $F_{A}(S)$ are not zero. We use this relationship and eliminate $C$ by substituting $C=1-A$ for the following analysis.

One plausible and straightforward interpretation of $B$ is as misfolded or mistranslated proteins that are produced erroneously during the replication of component A. Such waste molecules often aggregate with other molecules [26-28]. Alternatively, B components can be specific molecules such as HPF and YfiA [29-31], which inhibit catalytic activity by reacting with component A.

With regards to the formation of error or "waste" proteins, there are generally intracellular processes for reducing their fraction. These include kinetic proofreading, molecular chaperones, and protease systems. These error-correction or maintenance systems are energy demanding and require the nonequilibrium flow of

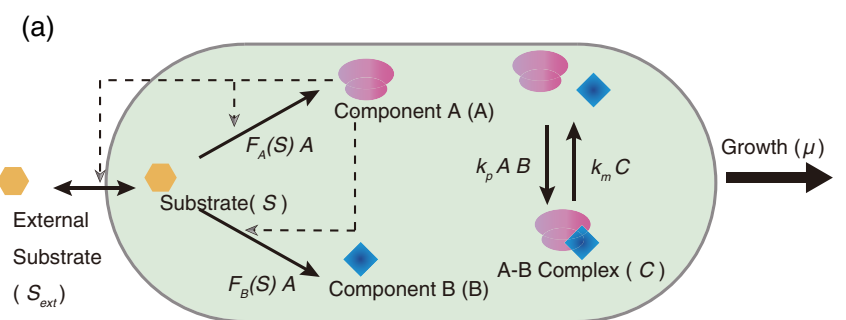

(b)

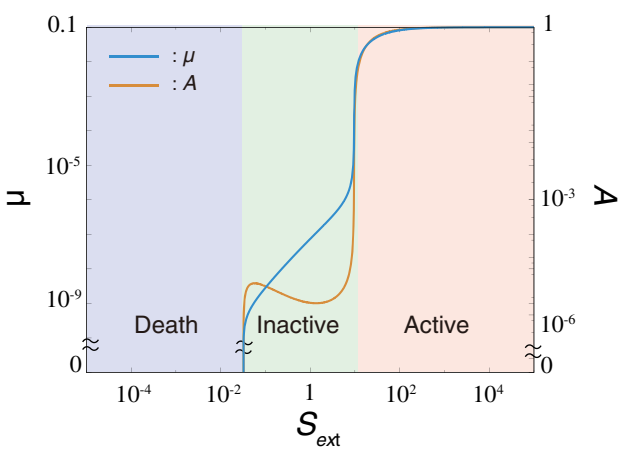

(c)

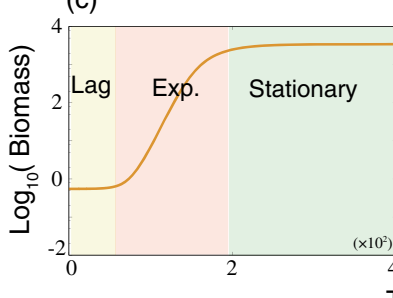

(d)

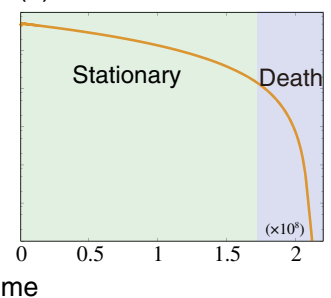

FIG. 1. (a) Schematic representation of the components and reactions in the present model. The concentration of each chemical changes according to the listed reactions. In addition, chemicals are spontaneously degraded at a low rate, and they become diluted because of the volume expansion of the cell. (b) Steady growth rate and the concentration of component A are plotted as functions of the external concentration of the substrate. $(\mathrm{c}, \mathrm{d})$ Growth curve of the model. Parameters are set as follows: $v=0.1, k_{p}=1.0, k_{m}=10^{-6}, K=1.0, K_{t}=10.0, d_{R}=d_{B}=10^{-5}$, $d_{C}=10^{-12}$. The detailed numerical method for panels (c) and (d) is given in Appendix C.

substrates [32,33]. Therefore, the performance of these mechanisms is inevitably reduced in a substrate (energy source)-poor environment. Thus, it naturally follows that the ratio of the synthesis of active proteins to wastes is an increasing function of the substrate concentration, i.e., $[d /(d S)]\left\{\left[F_{A}(S)\right] / F_{B}(S)\right\}>0$. In the present model, we assume that this ratio increases with the concentration and becomes saturated at higher concentrations, as in Michaelis-Menenten's form, and we choose $F_{A}(S)=[v S /(K+S)]\left[S /\left(K_{t}+S\right)\right]$ and $F_{B}(S)=$ $[v S /(K+S)]\left[K_{t} /\left(K_{t}+S\right)\right]$, for example.

Note that almost all the results presented in this article are obtained as long as $F_{A} \gg F_{B}$ holds for the nutrient-rich condition and $F_{A} \ll F_{B}$ for the nutrient-poor condition (see Sec. II G and Ref. [34]). Under this condition, a specific choice of the form of $F_{A}$ and $F_{B}$ is not important. 
This $S$ dependence of $F_{A} / F_{B}$ would be biologically plausible for the interpretations of component B as specific inhibitory proteins or "waste" (mistranslation) proteins. For the first interpretation, such proteins related with the stationary phase (HPF, YfiA, and others) are induced under stress conditions such as starvation $[29,30,35,36]$; thus, it is suggested that $F_{A} \gg F_{B}\left(F_{A} \ll F_{B}\right)$ for a large (small) amount of $S$, respectively. On the other hand, by adopting the latter (waste) interpretation, we derive $F_{A}(S)$ and $F_{B}(S)$ close to the above Michaelis-Menten's form, by considering a proofreading mechanism to reduce the mistranslation (see also Appendix B).

Here, we also note that, although the $S$ dependence of $F_{A} / F_{B}$ is relevant to derive quantitative laws on the lag time in agreement with experimental observation, it is not required just to show a transition to a suppressed growth state, as briefly discussed later (see Sec. III).

\section{RESULTS}

\section{A. Growth phases}

The steady state of the present model exhibits three distinct phases as a function of the external substrate concentration $S_{\text {ext }}$ [Fig. 1(b)], as computed by its steadystate solution. The three phases are distinguished by both the steady growth rate and the concentration of component A, which are termed as the active, inactive, and death phases, as shown in Fig. 1, whereas the growth rate shows a steep jump at the boundaries of the phases. The phases are characterized as follows. (i) In the active phase, the highest growth rate is achieved, where there is an abundance of component A molecules, which work freely as catalysts. (ii) In the inactive phase, the growth rate is not exactly zero but is drastically reduced by several orders of magnitude compared with that in the active phase. Here, almost all of the component $\mathrm{A}$ molecules are arrested through complex formation with component $\mathrm{B}$, and their catalytic activity is inhibited. (iii) At the death phase, a cell cannot grow, and all of the components A, B, and complexes go to zero. In this case, the cell goes beyond the so-called "point of no return" and can never grow again, regardless of the amount of increase in $S_{\text {ext }}$, since the catalysts are absent in any form. (As will be shown below, the active and inactive phases correspond to the classic exponential and stationary phases; however, to emphasize the single-cell growth mode, we adopt these former terms for now.)

The transition from the active to the inactive phase is caused by the interaction between components $\mathrm{A}$ and $\mathrm{B}$. In the substrate-poor condition, the amount of component $\mathrm{B}$ exceeds the total amount of catalytic proteins $(A+C)$, and any remaining free component $\mathrm{A}$ vanishes. Below the transition point from the inactive phase to the death phase, the spontaneous degradation rate surpasses the synthesis rate, at which point all of the components decrease. This transition point is simply determined by the balance condition $F_{A}=d_{A}$. Hence, if $d_{A}$ is set to zero, the inactive-death transition does not occur.

We now consider the time series of biomass (the total amount of macromolecules) that is almost proportional to the total cell number, under a condition with a given finite resource, which allows for direct comparison with experimental data obtained in a batch culture condition [Figs. 1(c) and 1(d)]. To compute the time series of biomass, we used a model including the dynamics of $S_{\text {ext }}$ in addition to $S, A, B$, and $C$. Details of this model are shown in Appendix C. In the numerical simulation, the condition with a given finite amount of substrates corresponding to the increase of cell number is implemented by introducing the dynamics of the external substrate concentration into the original model. Here, $S_{\mathrm{ext}}$ is decreased as the substrates are replaced by the biomass, resulting in cell growth. At the beginning of the simulation, the amount of biomass (i.e., cell number) stays almost constant and then gradually starts to increase exponentially. After the phase of exponential growth, the substrates are consumed, and the biomass increase stops. Then, over a long time span, the biomass stays at a nearly constant value until it begins to slowly decrease. Finally, the degradation dominates, and the biomass (cell number) falls off dramatically.

These successive transitions in the growth of biomass [Figs. 1(c) and 1(d)] from the initially inactive phase to the active, inactive, and death phases correspond to those observed among the lag, exponential, stationary, and death phases. As the initial condition was chosen as the inactive phase under a condition of rich substrate availability, most of the component A molecules are arrested in a complex at this point. Therefore, at the initial stage, dissociation of the complex into component A and component B progresses, and biomass is barely synthesized, even though a sufficient and plentiful amount of substrate is available. After the cell escapes this waiting mode, catalytic reactions driven by component A progress, leading to an exponential increase in biomass. Subsequently, the external substrate is depleted, and cells experience another transition from the active to the inactive phase. At this point, the biomass only decreases slowly, owing to the remaining substrate and the stability of the complex. However, after the substrate is depleted and components $\mathrm{A}$ and $\mathrm{B}$ are dissociated from the complex, the biomass decreases at a much faster rate, ultimately entering the death phase.

In the active phase with exponential growth, the present model exhibits classical growth laws, namely, (i) Monod's growth law and (ii) the growth rate vs ribosome fraction (see Fig. 6).

\section{B. Lag-time dependency on starvation time $T_{\text {stv }}$ and maximum growth rate $\mu_{\max }$}

In this section, we uncover the quantitative relationships among the basic quantities characterizing the transition between the active and inactive phases: i.e., lag time, 
starvation time, and growth rates. We demonstrate that the theoretical predictions agree well with experimentally observed relationships.

First, we compute the dependence of lag time $(\lambda)$ on starvation time $\left(T_{\text {stv }}\right)$. Up to time $t=0$, the model cell is set in a substrate-rich condition, $S_{\text {ext }}=S_{\text {ext }}^{\text {rich }}$, and it stays at a steady state with exponential growth. Then, the external substrate is depleted to $S_{\mathrm{ext}}=S_{\mathrm{ext}}^{\text {poor }}$ instantaneously. The cell is exposed to this starvation condition up to starvation time $t=T_{\text {stv }}$. Subsequently, the substrate concentration $S_{\text {ext }}$ instantaneously returns to $S_{\mathrm{ext}}^{\text {rich }}$. After the substrate level is recovered, it takes a certain amount of time for a cell to return to its original growth rate (Fig. S1 of Ref. [34]), which is the lag time $\lambda$ following the standard definition of lag time as the time period before the specific growth rate reaches its maximum value introduced by Penfold and Pirt [37,38]. Given this, the dependence of $\lambda$ on the starvation time $T_{\text {stv }}$ can be computed.

Next, we compute the dependence of the lag time $\lambda$ on $\mu_{\max }$. We choose the steady-state solution of the cell model under $S_{\mathrm{ext}}=S_{\mathrm{ext}}^{\mathrm{poor}}$ as the initial condition and compute the lag time $\lambda$ under the $S_{\text {ext }}=S_{\text {ext }}^{\text {rich }}$ condition against different values of $\mu_{\max }(=v)$ (following the standard method to measure the relationship between $\lambda$ and $\mu_{\max }$ [39]).

\section{Relationship between lag time and starvation time: $\lambda \propto \sqrt{T_{\text {stv }}}$}

We found that $\lambda$ increases in proportion to $\sqrt{T_{\mathrm{stv}}}$, as shown in Fig. 2(a). For comparison, the experimentally observed relationship between $\lambda$ and $T_{\text {stv }}$ is also plotted in Fig. 2(b), using reported data $[12,18,40]$ that also exhibited $\lambda \propto \sqrt{T_{\text {stv }}}$ dependence. Although this empirical dependence has been discussed previously [12], its theoretical origin has not been uncovered thus far.

Indeed, the origin of $\lambda \propto \sqrt{T_{\text {stv }}}$ can be explained by noting the anomalous relaxation of the component $\mathrm{B}$ concentration, which is caused by the interaction between components A and B. A general description of this explanation is given below, and the analytic derivation is given in Ref. [34].

First, consider the time series of chemical concentrations during starvation. In this condition, cell growth is inhibited by two factors: substrate depletion and inhibition of the catalytic activity of component A. Following the decrease in uptake due to depletion of $S_{\text {ext }}$, the concentration of $S$ decreases, resulting in a change in the balance between $\mathrm{A}$ and $\mathrm{B}$ (hereafter, we adopt the notation such that $A, B$, and $C$ also denote the concentrations of the corresponding (a)

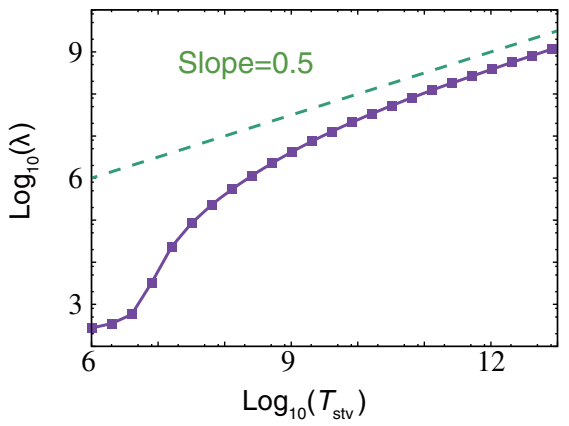

(c)

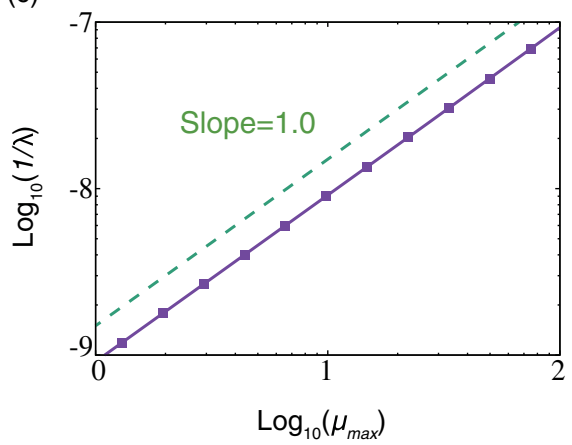

(b)

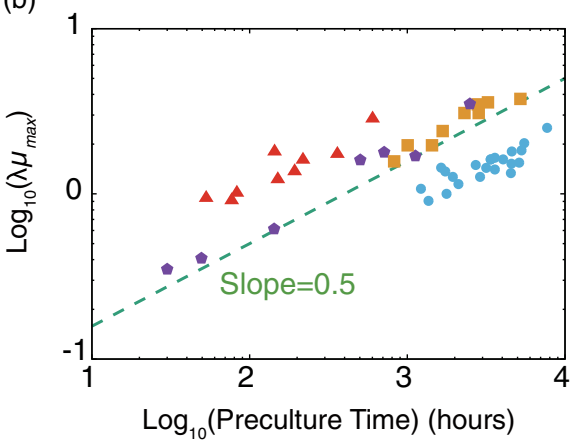

(d)

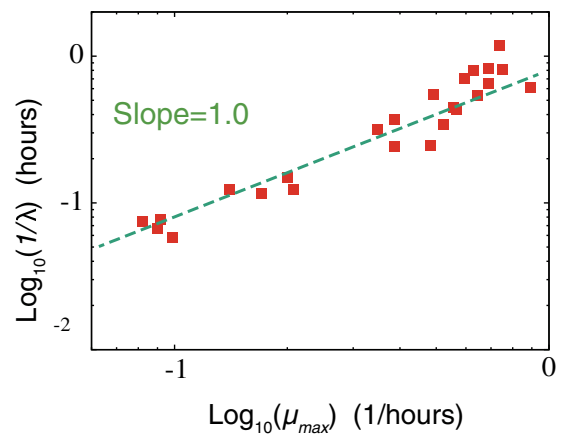

FIG. 2. (a,b) Lag time as a function of (a) starvation time or (b) preincubation time. The lag time is scaled by the maximum growth rate (inversely proportional to the shortest doubling time in the substrate-rich condition). Purple pentagons, cyan dots, and orange squares are adopted from Figs. 3, 6(a), and 6(b) of Augustin et al. [12], respectively, and the red triangles are extracted from the data in Table 1 of Pin et al. [40]. (c,d) Relationship between the lag time and maximum specific growth rate $\mu_{\max }$. Data are adopted from Table 1 of Oscar [39]. Parameters were set as follows: $S_{\mathrm{ext}}^{\mathrm{rich}}=10^{4}, S_{\mathrm{ext}}^{\mathrm{poor}}=10^{-2}, v=0.1, k_{p}=1.0, k_{m}=10^{-6}, K=1.0, K_{t}=10.0$, and $d_{A}=d_{B}=d_{C}=0$ (the same parameter values as in Fig. 1 except $d_{i}$ s). The lag time is computed as the time needed to reach the steady state under the $S_{\text {ext }}=S_{\text {ext }}^{\text {rich }}$ condition from an initial condition in the inactive phase. In panel (c), it is obtained by varying $v\left(=\mu_{\max }\right)$. 
chemicals). Under the $S_{\mathrm{ext}}^{\text {poor }}$ condition, the ratio of the synthesis of $\mathrm{B}$ to $\mathrm{A}$ increases. With an increase in $B, A$ decreases because of the formation of a complex with $\mathrm{B}$. Over time, more A becomes arrested, and the level of inactivation increases with the duration of starvation.

In this scenario, the increase of the concentration of B is slow. Considering that the complex formation reaction $A+B \leftrightarrow C$ rapidly approaches its equilibrium, i.e., $k_{p} A B \sim k_{m} C$, then $A$ is roughly proportional to the inverse of $B$ (recall $A+C=1$ ) if $B$ is sufficiently large. Accordingly, the synthesis rate of $\mathrm{B}$, given by $F_{B}(S) A$, is inversely proportional to its amount, i.e.,

$$
\dot{B}(t) \propto F_{B}(S) / B,
$$

and thus,

$$
d B^{2} / d t \sim \text { const. }
$$

Hence, the accumulation of component B progresses with $B(t) \propto \sqrt{t}$. (Note that because of $S$ depletion, the dilution effect is negligible).

Next, we consider the time series for the resurrection after recovery of the external substrate. During resurrection, $A$ is increased while $B$ is reduced. Since component $\mathrm{A}$ is strongly inhibited after starvation, the dilution effect from cell growth is the only factor contributing to the reduction of $B$. Noting that $\mu=F_{A} A$ and $A \propto 1 / B$, the dilution effect is given by $\mu B=F_{A} A B \propto B / B=$ const at the early stage of resurrection. Thus, the resurrection time series of $B$ is determined by the dynamics

$$
\dot{B}(t) \propto-\text { const },
$$

leading to the linear decrease of $B$, i.e., $B(t) \sim$ $B(0)-$ const $\times t$.

Let us briefly recapitulate the argument presented so far. The accumulated amount of component $\mathrm{B}$ is proportional to $\sqrt{T_{\text {stv }}}$, whereas during resurrection, the dilution of $\mathrm{B}$ progresses linearly with time, which is required for the dissociation of the complex of $\mathrm{A}$ and $\mathrm{B}$, leading to growth recovery. By combining these two estimates, the lag time satisfies $\lambda \propto \sqrt{T_{\text {stv }}}$.

\section{Relationship between the lag time and maximal growth rate: $\lambda \propto 1 / \mu_{\max }$}

The relationship $\lambda \propto 1 / \mu_{\max }$ is obtained by numerical simulation of our model, in line with experimental results [39] [Figs. 2(c) and (d)].

The relationship $\lambda \propto 1 / \mu_{\max }$ can also be explained by the characteristics of the resurrection time series. The dilution rate of $\mathrm{B}$ over time is given by $\mu B$, as mentioned above; thus, at the early stage, $\dot{B} \sim-\mu B$. In the substrate-rich condition, the substrate abundances are assumed to be saturated, so

$$
\lim _{S_{\mathrm{ext}}^{\text {rich } \rightarrow \infty}} \dot{B} \sim \lim _{S_{\mathrm{ext}}^{\text {irc }} \rightarrow \infty} F_{A} \cdot B / B=\mu_{\max }
$$

holds because $\lim _{S \rightarrow \infty} F_{A}(S)=\mu_{\max }$ is satisfied. Thus, it follows that $\lambda \propto 1 / \mu_{\max }$.

We also obtained an analytic estimation of the lag time as

$$
\lambda \sim \frac{1}{\mu_{\max }} \sqrt{2 F_{B} k_{p} / k_{m} T_{\mathrm{stv}}}
$$

(see Ref. [34] for conditions and calculations). In this form, the two relationships $\lambda \propto \sqrt{T_{\text {stv }}}$ and $\lambda \propto 1 / \mu_{\max }$ are integrated.

\section{E. Dependence of lag time on the starvation process}

So far, we have considered the dependence of lag time on the starvation time. However, in addition to the starvation period, the starvation process itself, i.e., the speed required to reduce the external substrate, has an influence on the lag time.

For this investigation, instead of the instantaneous depletion of the external substrate, its concentration is instead gradually decreased over time in a linear manner over the span $T_{\text {dec }}$, in contrast to the previous simulation procedure, which corresponds to $T_{\mathrm{dec}}=0$. Then, the cell is placed under the substrate-poor condition for the duration $T_{\text {stv }}$ before the substrate is recovered, and the lag time $\lambda$ is computed [41].

The dependence of the lag time $\lambda$ on $T_{\mathrm{stv}}$ and $T_{\mathrm{dec}}$ is shown in Fig. 3(a). While $\lambda$ monotonically increases against $T_{\text {stv }}$ for a given $T_{\text {dec }}$, it shows a drastic dependence on $T_{\text {dec }}$. If the external concentration of the substrate is reduced quickly (i.e., a small $T_{\text {dec }}$ ), the lag time is rather small. However, if the decrease in the external substrate concentration is slow (i.e., a large $T_{\mathrm{dec}}$ ), the lag time is much longer. In addition, this transition from a short to long lag time is quite steep.

The transition against the time scale of the environmental change manifests itself in the time series of chemical concentrations [see Fig. 3(b)]. With rapid environmental change, $S$ decreases first, whereas with slow environmental change, component A decreases first. In addition, the value of component B is different between the two cases, indicating that the speed of environmental change affects the degree of inhibition, i.e., the extent to which component $\mathrm{A}$ is arrested by component $\mathrm{B}$ to form a complex.

Now, we provide an intuitive explanation for two distinct inhibition processes. When $S_{\text {ext }}$ starts to decrease, a cell is in the active phase in which $\mathrm{A}$ is abundant. If the environment changes sufficiently quickly, there is not enough time to synthesize chemicals A or B because of the lack of $\mathrm{S}$, and the concentrations of chemical species are frozen near the initial state with abundant A. However, if the rate of environmental change is slower than that of the chemical reaction, the concentration of $\mathrm{B}(\mathrm{A})$ increases 


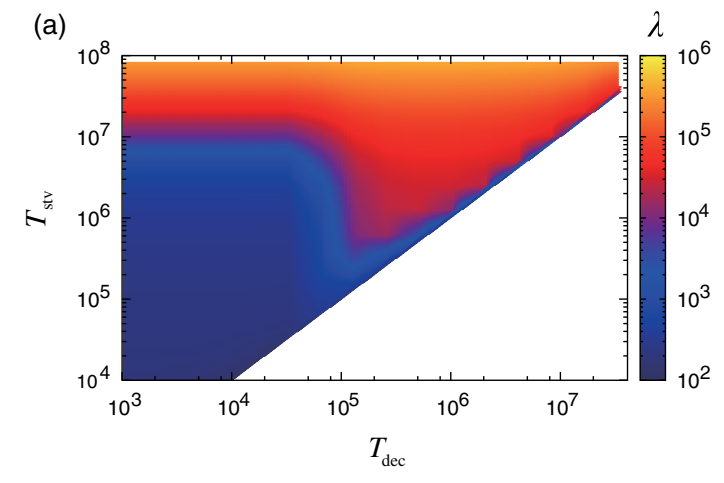

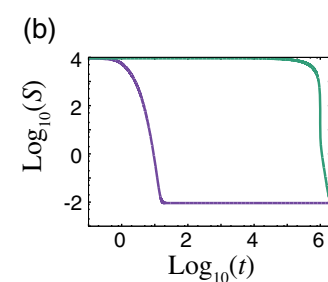

(d)

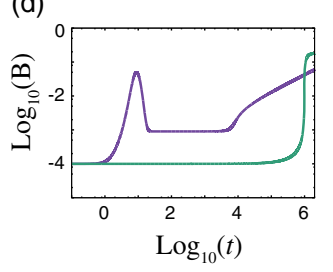

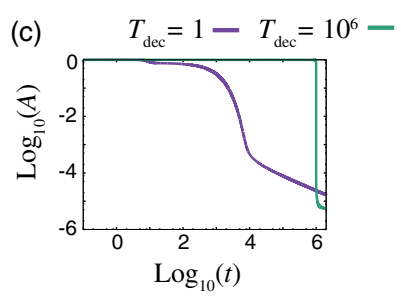

(e)

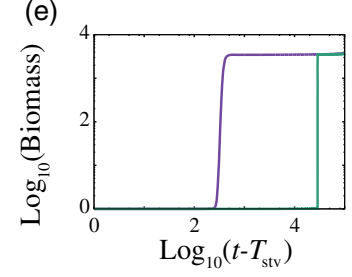

FIG. 3. (a) Dependence of lag time $\lambda$ on the time required to decrease the substrate $T_{\text {dec }}$ and starvation time $T_{\text {stv }}$. (b-d) Time series of starvation for different $T_{\mathrm{dec}}\left[T_{\mathrm{dec}}=10^{6}\right.$ (green line) and $T_{\mathrm{dec}}=1.0$ (purple line)] values-the internal concentrations of substrate $S$ (b), component A (c), and component B (d). (e) Time series of biomass during resurrection. The same parameter values as indicated in Fig. 2 were adopted. The batch culture model (which is used to compute a bacterial growth curve) was adopted to compute the time series of biomass accumulation (e). The time series of $\mu$ is shown in Fig. S3 of Ref. [34].

(decreases). Hence, A remains rich in the case of fast environmental change, whereas $\mathrm{B}$ is rich for a slow environmental change. In the former case, when the substrate is increased again, component $\mathrm{A}$ molecules are ready to work, so the lag time is short, which can be interpreted as a kind of "freeze-dry" process. Note that the difference in chemical concentration caused by different $T_{\mathrm{dec}}$ values is maintained for a long time because, in the case of slow (fast) environmental change, chemical reactions are almost completely halted because of the decrease of $A(S)$. Thus, the difference of lag time remains even for large $T_{\text {stv }}$, as shown in Fig. 3(a).

This lag time difference can also be explained from the perspective of dynamical systems [42]. For a given $S$, the temporal evolution of $A$ and $B$ is given by the flow in the state space of $(A, B)$. Examples of the flow are given in Fig. 4. The flow depicts $(d A / d t, d B / d t)$, which determines the temporal evolution. The flow is characterized by $A$ - and $B$-nullclines, which are given by the curves satisfying $d A / d t=0$ and $d B / d t=0$, as plotted in Fig. 4.

Note that at a nullcline, the temporal change of one state variable (either $A$ or $B$ ) vanishes. Thus, if two nullclines approach each other, then the time evolution of both concentrations $A$ and $B$ slow down, and the point where two nullclines intersect corresponds to the steady state. As shown in Fig. 4, nullclines come close together under the substrate-depleting condition, which provides a dynamical system account of the slow process in the inactive phase discussed so far.

For a fast change [i.e., small $T_{\text {dec }}$, Fig. 4(a)], $S$ is quickly reduced at the point where the two nullclines come close together. First, $B$ reaches the $B$ nullcline quickly. Then, the state changes along the almost-coalesced nullclines where the dynamics slow down. Thus, it takes a long time to decrease the $A$ concentration, so at the resumption of the substrate, a sufficient $A$ can be utilized.
In contrast, for a slow change (i.e., large $T_{\mathrm{dec}}$ ), the flow in $(A, B)$ gradually changes as shown in Figs. 4(b)-4(d). Initially, the state $(A, B)$ stays at the substrate-rich steady state. Because of the change in substrate concentration, two nullclines moderately move and interchange their vertical locations. Since the movement of nullclines is slow, the decrease in A progresses before the two nullclines come close together (i.e., before the process is slowed down). The temporal evolution of $A$ and $B$ is slowed down only after this decrease in $A$ [Figs. 4(c) and 4(d)]. Hence, the difference between cases with small and large $T_{\text {dec }}$ is determined according to whether the nullclines almost coalesce before or after the $A$ decrease, respectively.

These analyses allow us to estimate the critical time for a substrate decrease $T_{\mathrm{dec}}^{*}$ beyond the point at which $\lambda$ increases dramatically. The value of a fixed point $\left(A_{\mathrm{st}}, B_{\mathrm{st}}\right)$ depends on the substrate concentration, which drastically changes at the active-inactive transition point. If the relaxation to the fixed point is faster than the substrate decrease $T_{\mathrm{dec}}$, the system changes "adiabatically" to follow the fixed point at each substrate time during the course of a "slow decrease." The relaxation time is estimated by the smallest eigenvalue around the fixed point at the transition point. In the $k_{m} \rightarrow 0$ limit, this eigenvalue is equal to the growth rate at the active-inactive transition point. Since it is inversely proportional to $v$, the critical time $T_{\mathrm{dec}}^{*}$ for the substrate decrease is estimated as $T_{\mathrm{dec}}^{*} \propto 1 / v$. This dependence was also confirmed numerically (see Fig. S4 in Ref. [34]).

\section{F. Distribution of lag time}

So far, we have considered the average change of chemical concentrations using the rate equation of chemical 


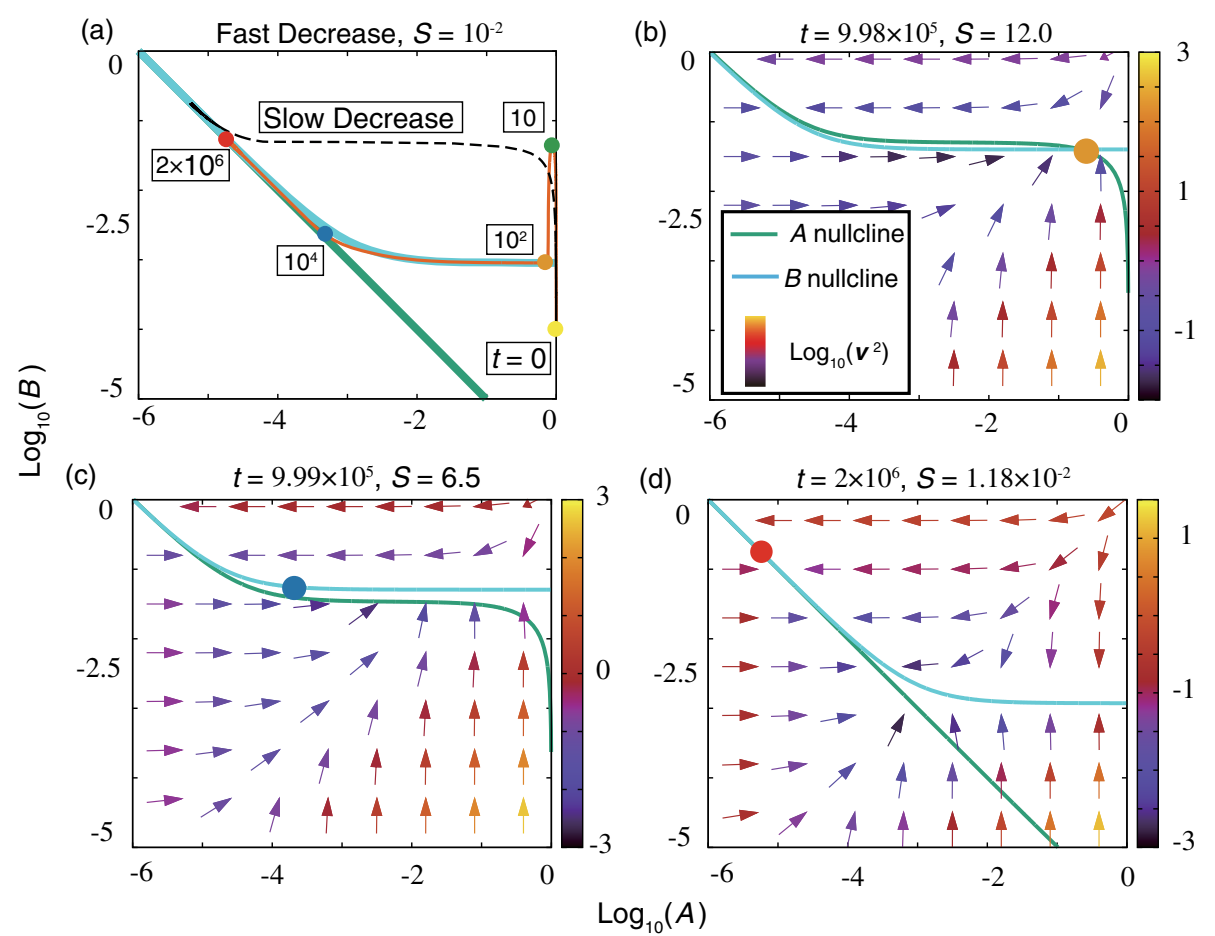

FIG. 4. Movement of nullclines and time evolution of state variables [circles within the state space $(A, B)$ ]. (a) The case of a fast substrate decrease (the orange line indicates the orbit, and numbers in white boxes indicate the time points). The orbit of a slow substrate decrease is also plotted (black dashed line). (b-d) The case of a slow substrate decrease. Each point is the value of the state variable at the indicated time and substrate concentration. The vector field $v=(d A / d t, d B / d t)$ is also depicted. Parameters are identical to those described in Fig. 2.

reactions. However, a biochemical reaction is inherently stochastic; thus, the lag time is accordingly distributed. This distribution was computed by carrying out a stochastic simulation of chemical kinetics using the Gillespie algorithm [43].

By increasing the starvation time, two types of lag-time distributions are obtained: (1) a skewed type and (2) a skewed type with an exponential long-time-tail type. Each distribution type changes as follows: First, when the starvation time is sufficiently long, the system enters the phase with the slow accumulation of B. Here, the relaxation is anomalous, leading to a skewed-type distribution. This skewed distribution is understood as follows. The number of component A molecules among cells takes on a Gaussian-like distribution just before the recovery of the external substrate concentration [44], whereas the lag time $\lambda$ is proportional to $B$ and thus to $1 / A$, as discussed in the last section. Then, the lag-time distribution $\lambda$ is obtained as the transformation of $1 / A \rightarrow \lambda$ from the Gaussian distribution of component $A$. This results in a skewed distribution with a long time tail as shown in Fig. 5(a). Second, when the starvation time is too long, the decrease in $A$ comes to the stage where its molecular number reaches 0 or 1. This results in a long time tail in the distribution. This effect occurs when the number of component A molecules becomes zero because it is inhibited by component $\mathrm{B}$. When the number of component $\mathrm{A}$ molecules becomes zero, the only reaction that can take place is a dissociation reaction $(C \rightarrow A+B)$. Since we assume that the time evolution of molecule numbers follows a Poisson process, the queueing time of dissociation obeys an exponential distribution Prob (queueing time $=t) \sim N_{C} k_{m} \exp \left(-N_{C} k_{m} t\right.$ ), where $N_{C}$ is the number of complexes formed. This exponential distribution is added to the skewed distribution, resulting in a long tail.

The distributions of the two cases are plotted in Fig. 5, together with experimental data adopted from Ref. [18]. The skewed distribution fits the experimental observations for the 0-day starvation data, whereas the distribution including the exponential tail is a good fit to the 1-day, 2-day, and 3-day distributions.

Here, each kinetic parameter alters the critical starvation time around which the shape of the distribution starts to change; for example, a small $k_{m}$ makes it easier to obtain the type-three distribution. However, kinetic parameters do not change the shape of the distribution directly, as confirmed computationally.

The distribution of lag time was traditionally thought to follow the normal distribution $[8,47]$ until single-cell measurements were carried out for a long time span 
(a)

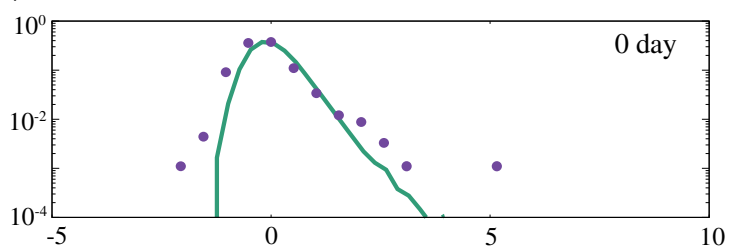

(b)

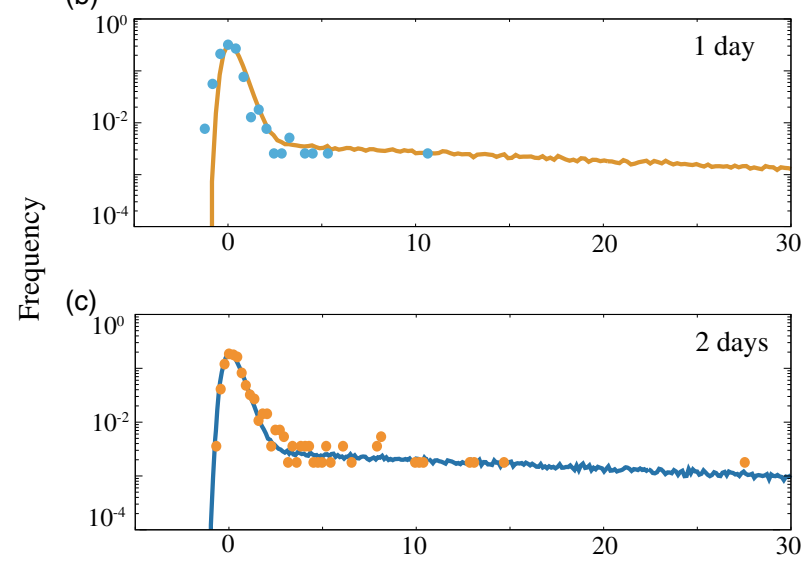

(d)

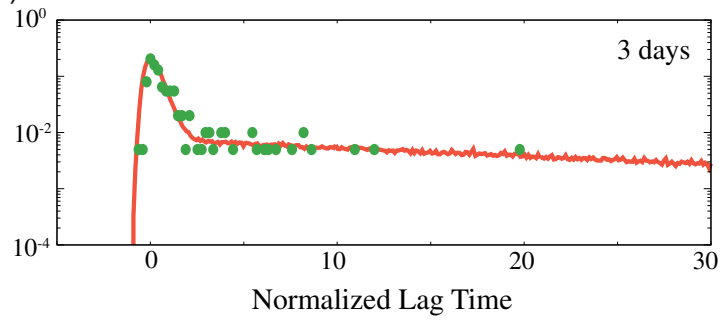

FIG. 5. Distribution of lag time obtained by model simulation (solid line) with experimental data (lag-time distribution of cultures starved for the indicated days) overlaid. The horizontal axis of each distribution was normalized by using its peak point (Peak) and the full width at half maximum (FWHM) as $\lambda \rightarrow(\lambda-$ Peak $) /$ FWHM. Experimental data were extracted from those presented in Fig. 1(e) of Reismann et al. [18]. Methods of stochastic simulations, the procedure used to compute Peak and FWHM, and parameter values are given in Appendix C.

[18]. The preset model also generates the normal distribution of lag time if the starvation time is too short, whereas the normal distribution of lag time in earlier experiments would originate from the limitation of experimental procedures. For example, a cell that regains growth in a colony ends up dominating the colony; thus, the fluctuation of the shortest lag time governs the behavior. However, identification of a small fraction of bacteria with a long lag time is difficult, owing to the limited capacity of cell tracking (as indicated in Ref. [18]).

\section{G. Remarks on the choice of parameters to fit the experimental data}

Although there are several parameters in the model and the results depend on these values, the basic results on the active-inactive transition, suppression of growth, and quantitative relationships with lag time are obtained for a large parameter region. Conditions of the parameter values to obtain these main results are given in Ref. [34] and are summarized in Table I. Here, an important parameter is $k_{m}$, which we assumed to be the smallest among all other parameter values. This choice was made to facilitate analytic calculations, and the condition for $k_{m}$ can be relaxed. For example, we plotted the growth rate at the steady state in Fig. S5 of Ref. [34], indicating that the active-inactive transition occurs as long as $k_{m}<k_{p}$ holds.

Next, we estimate realistic parameter values such as the value of $v$ from the literature. However, several parameter values could not be estimated directly from experimentally reported data because this would require quantitative studies at the stationary phase, which are not currently available. Thus, we estimated other parameter values by fitting Monod's growth law [1], as well as by using the reported relationship between the ribosome fraction and growth rate [4,48,49] (Fig. 6) [50]. Since the number of parameters is greater than the minimum number required to fit the two laws in Fig. 6, the choice of parameter values is not unique. A possible set of parameter values is listed in Table II in Appendix D.

In fitting the two growth laws in Fig. 6, we have also found that $v$ is proportional to the maximum growth rate and that it negatively correlates with the slope of the linear relationship between ribosome fraction and growth rate, while $r$ (the fraction of actively translating ribosome) decreases and $k_{p}$ increases the $y$ offset of the linear relation, respectively [51].

TABLE I. Predictions and assumptions.

\begin{tabular}{|c|c|c|}
\hline Result & Assumption & Condition (Prediction) \\
\hline Active-inactive transition point & $k_{m} \sim 0$ & $1+2 F_{A}\left(S_{\mathrm{st}}\left(S_{\mathrm{ext}}^{\mathrm{act}-\text { inact }}\right)\right) / k_{p}=\sqrt{1+4 F_{B}\left(S_{\mathrm{st}}\left(S_{\mathrm{ext}}^{\text {act-inact }}\right)\right) / k_{p}}$ \\
\hline Inactive-death transition point & - & $F_{A}\left(S_{\mathrm{st}}\left(S_{\mathrm{ext}}^{\text {inact-death }}\right)\right)=d_{A}$ \\
\hline Analytic estimation of lag time & $\begin{array}{l}k_{m} \sim 0, \text { dynamics } \\
\text { of } S \text { is faster than }(A, B)\end{array}$ & $\lambda \sim\left\{1 /\left[F_{A}\left(S_{\mathrm{st}}\left(S_{\mathrm{ext}}^{\mathrm{rich}}\right)\right)\right]\right\} \sqrt{2 F_{B}\left(S_{\mathrm{st}}\left(S_{\mathrm{ext}}^{\mathrm{poor}}\right)\right) k_{p} / k_{m} T_{\mathrm{stv}}}$ \\
\hline $\begin{array}{l}\text { Contiguity of nullclines } \\
\text { (slow relaxation) }\end{array}$ & - & $\begin{array}{c}A_{\text {A-nullcline }}(B) \sim\left\{G(0, B) /\left[F_{A}(S)-G^{\prime}(0, B)\right]\right\} \\
A_{\text {B-nullcline }}(B) \sim\left\{G(0, B) /\left[F_{B}(S)-F_{A}(S) B-G^{\prime}(0, B)\right]\right\}\end{array}$ \\
\hline
\end{tabular}


(a)

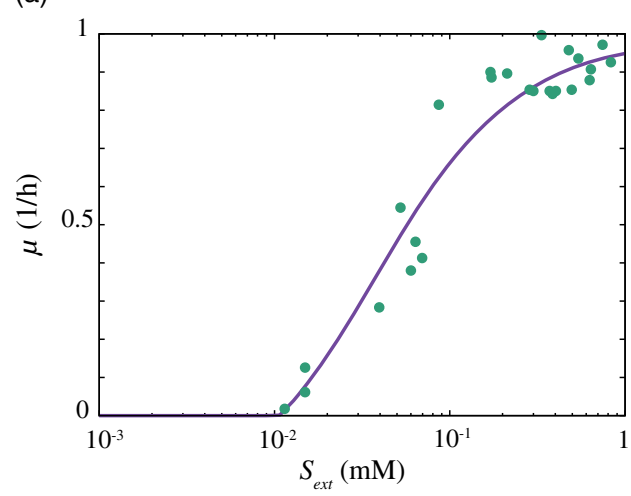

(b)

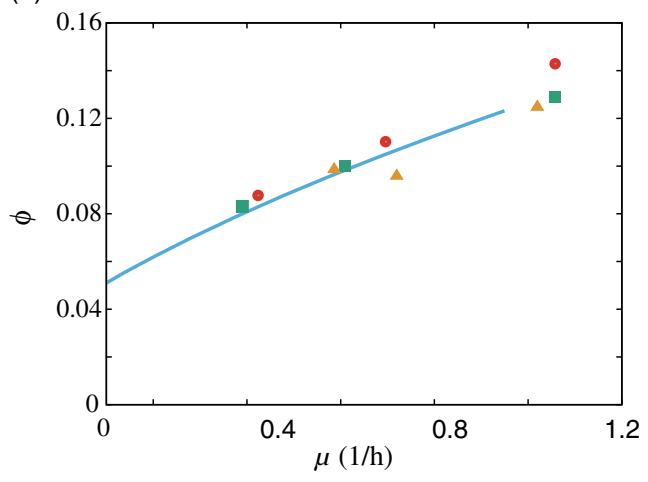

FIG. 6. Comparison of the model results using estimated values (Table II) with experimental values. (a) The specific growth rate is plotted as a function of the external substrate (glucose) concentration. Experimental data are adopted from Monod [1]. (b) Ratio of ribosomal proteins (component A) to total proteins as a function of the specific growth rate: Orange triangles are from Scott et al. [4], red circles are from Bremer and Dennis [48], and green squares are from Forchhammer et al. [49]. In panel (b), the theoretical curve from the model is plotted up to about 1.0 because we obtained the parameter values by fitting the $\mu-\phi$ relation and the Monod equation with the maximum growth rate of $\mu_{\max } \sim 1.0$.

\section{DISCUSSION}

Here, we developed a coarse-grained model consisting of a substrate, autocatalytic active protein (component A), a non-growth-facilitating component (component B), and an A-B complex, C. In the steady state, the model shows distinct phases, i.e., the active, inactive, and death phases. In addition, the temporal evolution of the total biomass is consistent with the bacterial growth curve. The present model not only satisfies the already-known growth laws in the active phase but also demonstrates two relationships, $\lambda \propto \sqrt{T_{\text {stv }}}$ and $\lambda \propto 1 / \mu_{\max }$, concerning the duration of the lag time $\lambda$. Although these two relationships have also been observed experimentally, their origins and underlying mechanisms had not yet been elucidated. The present model can explain these relationships based on the formation of a complex between components A and B, whose increase in the starvation condition hinders the catalytic reaction.

The above two laws are also generally derived for the inactive phase, which corresponds to the stationary phase, as long as the ratio of the synthesis of component $\mathrm{B}$ to that of component $\mathrm{A}$ is increased along with a decrease in the external substrate concentration. This condition can also be interpreted as a natural consequence of the waste-reducing (or error-correcting) process that is ubiquitous in a cell, which demands energy when assuming that component B consists of waste molecules. These laws are also derived if the waste is interpreted as a product of erroneous protein synthesis, where a proofreading mechanism to correct the error, which also requires energy, works inefficiently in a substrate-poor condition. The inhibition of growth by waste proteins is experimentally discussed by Nucifora and others [26-28]. Aggregation of such waste proteins can inhibit the catalytic activity of proteins, although its role in the transition to the inactive phase remains to be elucidated. Alternatively, instead of waste proteins, we can also interpret such nonautocatalytic proteins as specific inhibitory molecules binding ribosomes such as YfiA and HPF [29-31].

For a simpler model, one could eliminate the substrate dependence of $F_{B}(S) / F_{A}(S)$. Indeed, even in this simpler form, the active-inactive transition itself is observed if we finely tune the parameter values, as the decrease in substrate flow decreases the dilution, which in turn increases the fraction of complexes formed. Nevertheless, the accumulation of nonautocatalytic proteins is not facilitated with a substrate decrease, and the increase in the lag time as $\lambda \propto \sqrt{T_{\text {stv }}}$ does not follow. Hence, this simpler model will not be appropriate to explain the behavior of the present cells, although it might provide relevant insight as a general mechanism for the "inactive" or "dormancy" phase in the context of protocells.

Although the cell state with exponential growth has been extensively analyzed in previous theoretical models, the transition to the phase with suppressed growth has thus far not been theoretically explained. Our model, albeit simple, provides an essential and general mechanism for this transition with consideration of the complex formation between components $\mathrm{A}$ and $\mathrm{B}$, which can be experimentally tested.

The model here may also be relevant to study growth arrest such as stringent response [54,55]. In this case, ppGpp, the effector molecule of the stringent response, is known to destabilize the open complex of all promoters, causing the global reduction of macromolecular synthesis, playing a similar role as component $\mathrm{B}$ in the present paper [56-59]. Additionally, rpoS, the sigma factor of stationaryphase genes, lies downstream of ppGpp [60], and it is reported that the mutant lacking ppGpp (which might correspond to inhibition of the component B in our model) 
shows a physiological state reminiscent of exponentially growing bacteria even under starvation [61].

Moreover, the model predicts that the lag time differs depending on the rate of external depletion of the substrate, which can also be examined experimentally. Recently, the bimodal distribution of growth resumption time from the stationary phase was reported in a batch culture experiment [19]. The heterogeneous depletion of a substrate due to the spatial structure of a bacterial colony is thought to be a potent cause of this bimodality, and progress toward gaining a deeper understanding of this concept is underway. Since the present model shows different lag times for different rates of environmental change, it can provide a possible scenario to help explain this bimodality.

\section{ACKNOWLEDGMENTS}

The authors would like to thank S. Krishna, S. Semsey, N. Mitarai, A. Kamimura, N. Saito, and T. S. Hatakeyama for useful discussions, and I. L. Reisman, N. Balaban, and J. C. Augustin for providing data. This research is partially supported by the Platform for Dynamic Approaches to Living System from the Japan Agency for Medical Research and Development (AMED), Grant-in-Aid for Scientific Research (S) [No. 15H05746 from the Japan Society for the Promotion of Science (JSPS)], and JSPS Grant No. 16J10031.

\section{APPENDIX A: MODEL WITHOUT INTERACTION BETWEEN THE TWO COMPONENTS}

To clarify the necessity of the interaction between the two components to obtain the main results, we remove the complex formation between $\mathrm{A}$ and $\mathrm{B}$ (by setting $k_{p}$ and $k_{m}$ to zero). Then, the A-B complex is eliminated, and our model is given as

$$
\begin{aligned}
& \dot{A}=F_{A}(S) A-F_{A}(S) A^{2}-d_{A} A, \\
& \dot{B}=F_{B}(S) A-F_{A}(S) A B-d_{B} B .
\end{aligned}
$$

(We assume that the internal concentration of the substrate is equal to that of the external concentration of the substrate, and ignore the substrate dynamics.) The steady solution is

$$
A_{s t}=1-\frac{d_{A}}{F_{A}(S)}, \quad B_{s t}=F_{B}(S) \frac{1-d_{A} / F_{A}(S)}{F_{A}(S)-d_{A}+d_{B}},
$$

and the steady growth rate is given as $\mu_{s t}=F_{A}(S) A_{s t}=$ $F_{A}(S)-d_{A}$. Therefore, the present model without an interaction between components $\mathrm{A}$ and $\mathrm{B}$ exhibits only the active-death transition at $S^{*}$, satisfying $F_{A}\left(S^{*}\right)=d_{A}$. In addition, the dynamics of the system are calculated as

$$
\begin{aligned}
& A(t)=\frac{1}{1-\exp \left(-F_{A} t\right)\left(1-A(0)^{-1}\right)}, \\
& B(t)=\frac{F_{B}}{F_{A}} \frac{1-\exp \left(-F_{A} t\right)\left(1-\frac{B(0) F_{A}}{A(0) F_{B}}\right)}{1-\exp \left(-F_{A} t\right)\left(1-A(0)^{-1}\right)},
\end{aligned}
$$

where we neglect $d_{i}$, as in the main text. Therefore, if the model cell, Eq. (A2), restarts growth in a high- $S\left(S_{\text {rich }}\right)$ value environment after exposure to the starvation condition (low- $S$ value), $A(t)$ and $B(t)$ exponentially converge to the substrate-rich steady state. Hence, the time for growth recovery $T_{\text {rec }}$ is quite short, which is calculated as

$T_{\text {rec }}=\frac{1}{F_{A}\left(S_{\text {rich }}\right)} \ln \left(\left(\frac{B_{p}}{B_{r}}-1\right)\left(1-e^{-F_{A}\left(S_{\text {poor }}\right) T_{\text {stv }}}\right)\right)+$ const,

as a function of starvation time $T_{\mathrm{stv}}$. Here, $B_{p}$ and $B_{r}$ are the steady concentrations of component $B$ under the substrate-poor and substrate-rich environments, respectively. Obviously, this relationship is far from the relationship between lag and starvation time.

\section{APPENDIX B: REDUCTION OF THE KINETIC PROOFREADING MODEL}

In the main text, the concrete forms of $F_{A}$ and $F_{B}$ were predetermined by assuming the characteristic $[d /(d S)]\left(F_{A} / F_{B}\right)>0$, which is essential for the activeinactive transition. In this section, we show that this characteristic is derived from a simple polymer elongation model with a kinetic proofreading scheme [33] by assigning a correct polymer as $\mathrm{A}$ and an erroneous one as B. Indeed, $[d /(d S)]\left(F_{A} / F_{B}\right)>0$ originates from an error in the synthesis of component $\mathrm{A}$ that consequently inhibits the synthetic reactions.

Polymer elongation is essential to synthesize macromolecules. It is well known that ribosomes elongate a polypeptide chain following receipt of the information from messenger RNA. However, since the transfer RNA (tRNA) discrimination by a ribosome is not perfect, there is always a certain probability for mistranslation (i.e., the wrong choice of tRNA). Kinetic proofreading is one of the possible error-correction mechanisms in such a polymerization system, which demands energy. We find that the synthesis ratio of mistranslated proteins to a "correct" protein increases under the substrate-depleting condition.

For the polymerization reaction, we introduce two monomers, "correct" and "wrong" monomers, as simplified from real amino acids. In reality, there are 20 amino acids and one tRNA that specifies one amino acid, i.e., one correct and 19 wrong monomers with a certain affinity lower than that of the correct monomer.

In the model, a polymer is elongated up to the length $L$ with the aid of the catalytic activity of the "correct" protein, i.e., the ribosome. The matured polymer with length $L$ is 
spontaneously folded into a protein; the proteins consisting of only correct monomers are correct proteins with catalytic activity, whereas those with other monomer sequences turn into mistranslated proteins. The elongation process progresses under a kinetic proofreading mechanism (Fig. 7).
As in the original model, mistranslated proteins inhibit the correct protein's catalytic activity by forming a complex with it, while the growth is facilitated by the activity of correct proteins. The dynamics of the polymer elongation part are given by

$$
\begin{aligned}
\frac{d\left[A_{i}(x)\right]}{d t}=-\hat{k}_{0} \sum_{Y=C, D}\left(\left[A_{i}(x)\right]\left[M_{Y}\right]-\rho_{Y} \hat{l}_{0}\left[A_{i}(x) M_{Y}\right]\right)-\hat{k}_{2} \sum_{Y=C, D}\left(\left[A_{i}(x)\right]\left[M_{Y}\right]-\rho_{Y} \hat{l}_{2}\left[A_{i}(x) M_{Y}^{*}\right]\right)+\hat{v}\left[A_{i-1}\left(x^{-}\right) M_{\mathrm{ter}(x)}^{*}\right], & (1 \leq i \leq L-1), \\
\frac{d\left[A_{i}(x) M_{Y}\right]}{d t}= & \hat{k}_{0}\left(\left[A_{i}(x)\right]\left[M_{Y}\right]-\rho_{Y} \hat{l}_{0}\left[A_{i}(x) M_{Y}\right]\right)-\hat{k}_{1}\left(\left[A_{i}(x) M_{Y}\right] \alpha-\hat{l}_{1}\left[A_{i}(x) M_{Y}^{*}\right] \beta\right), \quad(0 \leq i \leq L-1), \\
\frac{d\left[A_{i}(x) M_{Y}^{*}\right]}{d t}= & \hat{k}_{1}\left(\left[A_{i}(x) M_{Y}\right] \alpha-\hat{l}_{1}\left[A_{i}(x) M_{Y}^{*}\right] \beta\right)+\hat{k}_{2}\left(\left[A_{i}(x)\right]\left[M_{Y}\right]-\rho_{Y} \hat{l}_{2}\left[A_{i}(x) M_{Y}^{*}\right]\right)-\hat{v}\left[A_{i}(x) M_{Y}^{*}\right] \quad(0 \leq i \leq L-1),
\end{aligned}
$$

where $\left[M_{C}\right]$ and $\left[M_{D}\right]$ denote the concentrations of correct and wrong monomers, respectively. Note that $\left[A_{i}(x)\right],\left[A_{i}(x) M_{Y}\right]$, and $\left[A_{i}(x) M_{Y}^{*}\right]$ represent the concentration of a complex of correct proteins and a polymer with length $i$, a correct protein-polymer-monomer complex, and an activated correct protein-polymer-monomer complex, respectively, where $x$ denotes a monomer sequence such as $C C D C \ldots$, with $C$ and $D$ indicating the correct and wrong monomers, respectively. Here, $\operatorname{ter}(x)$ and $x^{-}$indicate the last monomer $(C$ or $D)$ of a monomer sequence $x$ and the partial monomer sequence of $x$ from which the last monomer [i.e., $\operatorname{ter}(x)$ ] has been removed, respectively. Note that $\left[A_{0}\right]$ denotes the concentration of the correct protein; $\hat{v}$ and $\hat{k}_{i}$ 's are the rate constants of the chemical reactions; and the $\hat{l}_{i}$ 's are the Boltzman factors of each chemical reaction. We assume that dissociation of the matured polymer from correct proteins and polymer folding into proteins takes place instantaneously. Here, $\alpha$ and $\beta$ are the concentration energy currencies, for example, GTP and
GDP, respectively, and $\rho_{i}$ reflects the difference in affinity between the wrong monomer $(D)$ and the correct monomer (C) (we set $\rho_{C}$ as unity).

At the steady state, the synthesis rates of correct and mistranslated proteins, $J_{\mathrm{A}}^{L}$ and $J_{\mathrm{B}}^{L}$, are given by

$$
\begin{aligned}
& J_{\mathrm{A}}^{L}=\hat{v}\left[A_{0}\right]\left[M_{C}\right] H_{C} \Xi_{C}^{L-1}, \\
& J_{\mathrm{B}}^{L}=\hat{v}\left[A_{0}\right]\left(H_{C}\left[M_{C}\right]+H_{D}\left[M_{D}\right]\right)\left(\Xi_{C}+\Xi_{D}\right)^{L-1}-J_{\mathrm{A}}^{L},
\end{aligned}
$$

where functions $\Xi_{i}$ and $H_{i}$ are given by

$$
\begin{aligned}
\Xi_{i} & =\frac{\hat{v} H_{i}\left[M_{i}\right]}{\hat{k}_{0} \sum_{j}\left(1-\rho_{j} \hat{l}_{0} Z_{j}\left[M_{j}\right]\right)+\hat{k}_{2} \sum_{j}\left(1-\rho_{j} \hat{l}_{2} H_{j}\left[M_{j}\right]\right)}, \\
H_{i} & =\frac{\hat{k}_{2}\left(\hat{k}_{1} \alpha+\hat{k}_{0} \rho_{i} \hat{l}_{0}\right)+\hat{k}_{0} \hat{k}_{1} \alpha}{\left(\hat{k}_{1} \alpha+\hat{k}_{0} \rho_{i} \hat{l}_{0}\right)\left(\hat{k}_{1} \hat{l}_{1} \beta+\hat{k}_{2} \rho_{i} \hat{l}_{2}+\hat{v}\right)-\hat{k}_{1}^{2} \hat{l}_{1} \alpha \beta}, \\
Z_{i} & =\frac{\hat{k}_{0}+\hat{k}_{1} \hat{l}_{1} \beta H_{i}}{\hat{k}_{0} \rho_{i} \hat{l}_{0}+\hat{k}_{1} \alpha} .
\end{aligned}
$$

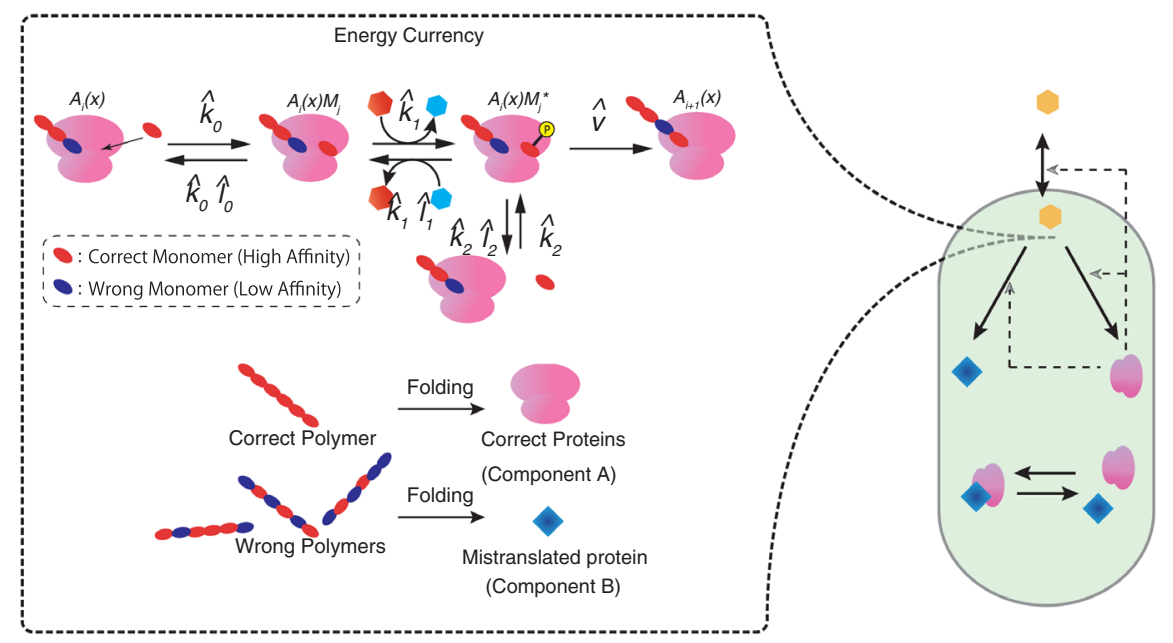

FIG. 7. Schematic representation of a polymer elongation system with kinetic proofreading. The reactions, other than the synthesis part $\left[F_{A}(S) A\right.$ and $\left.F_{B}(S) A\right]$, are identical to those of the original model (2). 
Here, $\Xi_{0}$ and $\Xi_{1}$ denote the rate of polymer elongation with the wrong and correct monomers, respectively. Now, we set the functional form of $\alpha$ and monomer concentrations $\left[M_{C}\right]$ and $\left[M_{D}\right]$ to obtain the concrete values of $J_{\mathrm{c}}^{L}$ and $J_{\mathrm{B}}^{L}$. It is natural to assume that $\alpha$ and $\left[M_{i}\right]$ are increasing functions of the internal substrate concentration $[S]$. Here, we adopt a Michaelis-Menten's type form $\alpha=[S] /\left(K_{a}+[S]\right), \beta=$ $K_{a} /\left(K_{a}+[S]\right)$, and $\left[M_{C}\right]=\left[M_{D}\right]=[M]_{\max }[S] /\left(K_{S}+[S]\right)$.

Although $J_{\mathrm{A}}^{L}([S])$ and $J_{\mathrm{B}}^{L}([S])$ do not completely agree with the form we adopted for $F_{A}(S) A$ and $F_{B}(S) A$ in the original model, the conditions discussed in Sec. II of Ref. [34] are nevertheless satisfied, as shown in Fig. 8.

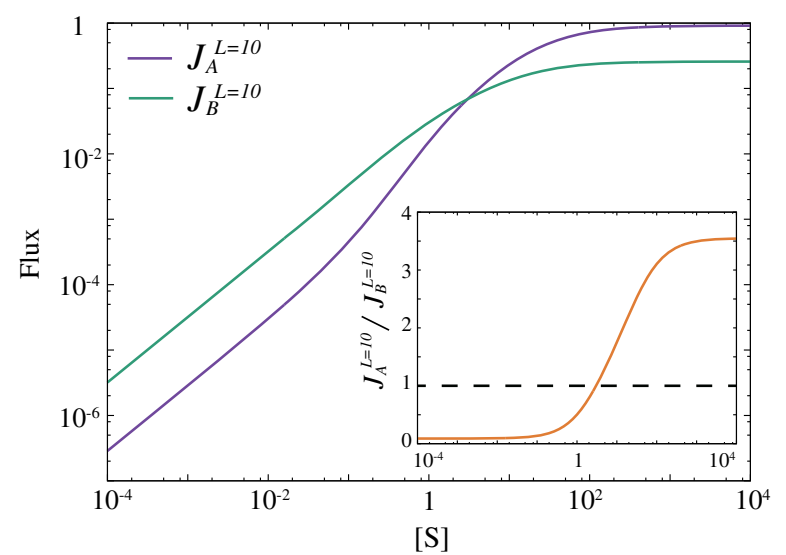

FIG. 8. $J_{\mathrm{A}}^{L=10}$ and $J_{\mathrm{B}}^{L=10}$ are plotted against the substrate concentration $[S]$. The ratio of $J_{\mathrm{A}}^{L=10}$ to $J_{\mathrm{B}}^{L=10}$ is also plotted in the inset of the figure. Parameters for the polymer elongation part are set to $\hat{v}=0.1, \rho_{C}=1.0, \rho_{D}=10.0, \hat{k}_{0}=10^{5}, \hat{k}_{1}=10^{2}$, $\hat{k}_{2}=10.0, \hat{l}_{0}=\hat{l}_{1}=\exp (-1), \hat{l}_{2}=\exp (1), K_{a}=10.0, K_{S}=1.0$, $[M]_{\max }=1.0$, and $\left[A_{0}\right]=1.0$.

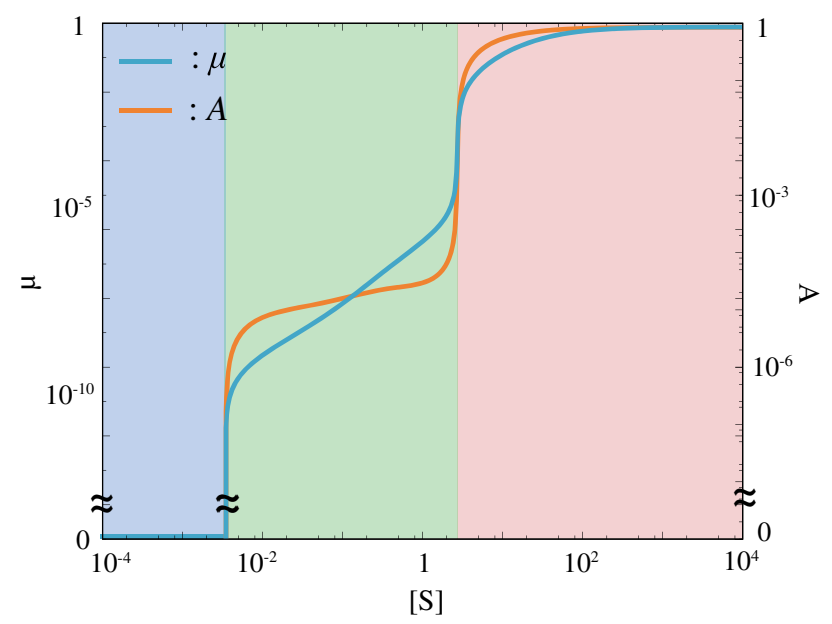

FIG. 9. Steady growth rate of the model with polymerization and kinetic proofreading. Here, $J_{\mathrm{A}}^{L=10}$ and $J_{\mathrm{B}}^{L=10}$ are adopted for the synthetic reaction rate of components A and B. Parameters for $J_{\mathrm{A}}^{L=10}$ and $J_{\mathrm{B}}^{L=10}$ are identical to those in Fig. 8, and others are set to be the same.
In particular, $\{(d) / d[S]\} J_{\mathrm{A}}^{L} / J_{\mathrm{B}}^{L}>0$ holds. Indeed, using this model, we obtained the same active, inactive, and death phases, as well as the same growth curve and other quantitative laws. As an example, Fig. 9 shows the steady growth rate as a function of the external substrate concentration $[S]_{\mathrm{ext}}$. Furthermore, for any $L$, the same behaviors are obtained, as $J_{\mathrm{A}}^{L}$ and $J_{\mathrm{B}}^{L}$ satisfy the condition outlined in Sec. II of Ref. [34]. It is also confirmed that the ratio of $J_{\mathrm{A}}^{L}([S])$ to $J_{\mathrm{B}}^{L}([S])$ increases as $[S]$ increases for any $L$.

\section{APPENDIX C: DETAILS OF MODELS AND SIMULATION PROCEDURES}

To obtain the growth curve shown in Figs. 1(c) and 1(d) in the main text, we added the dynamics of the substrates in the external environment, as well as cell volume growth. By representing the dynamics according to the amounts of chemicals rather than their concentrations, the model is given by

$$
\begin{aligned}
\frac{d N_{S_{\mathrm{ext}}}=}{d t} & -N_{A}\left(N_{S_{\mathrm{ext}}} / V_{\mathrm{bath}}-N_{S} / V\right), \\
\frac{d N_{S}}{d t}= & -F_{A}\left(N_{S} / V\right) N_{A}-F_{B}\left(N_{S} / V\right) N_{P} \\
& +N_{A}\left(N_{S_{\mathrm{ext}}} / V_{\mathrm{bath}}-N_{S} / V\right), \\
\frac{d N_{A}}{d t}= & F_{A}\left(N_{S} / V\right) N_{A}-k_{p} N_{A} N_{B} / V \\
& +k_{m} N_{C}-d_{A} N_{A}, \\
\frac{d N_{B}}{d t}= & F_{B}\left(N_{S} / V\right) N_{A}-k_{p} N_{A} N_{B} / V \\
& +k_{m} N_{C}-d_{B} N_{B}, \\
\frac{d N_{C}}{d t}= & k_{p} N_{A} N_{B} / V-k_{m} N_{C}-d_{C} N_{C}, \\
\frac{d V}{d t}= & F_{A}\left(N_{S} / V\right) N_{A},
\end{aligned}
$$

where $N_{S_{\text {ext }}}$ is the amount of substrate in the external environment at volume $V_{\text {bath }}$, and $N_{S}, N_{A}, N_{B}$, and $N_{C}$ are the amounts of each chemical within the cell at volume $V(t)$, respectively. Here, $V(t)$ is the volume of a cell. The dilution effect is introduced by dividing the amount of each chemical by $V(t)$, and $S_{\text {ext }}$ is the total amount of the external substrate contained in the culture system with volume $V_{\text {bath }}$ (set to unity). For all other parameters, the same values as shown in Fig. 1 were adopted.

To obtain the lag-time distribution, we performed a stochastic simulation. We computed the model equation according to the volume change 


$$
\begin{aligned}
\frac{d N_{S}}{d t}= & -F_{A}\left(N_{S} / V\right) N_{A}-F_{B}\left(N_{S} / V\right) N_{A} \\
& +N_{A}\left(N_{S_{\text {ext }}} / V_{\text {bath }}-N_{S} / V\right), \\
\frac{d N_{A}}{d t}= & F_{A}\left(N_{S} / V\right) N_{A}-k_{p} N_{A} N_{B} / V+k_{m} N_{C}, \\
\frac{d N_{B}}{d t}= & F_{B}\left(N_{S} / V\right) N_{A}-k_{p} N_{A} N_{B} / V+k_{m} N_{C}, \\
\frac{d N_{C}}{d t}= & k_{p} N_{A} N_{B} / V-k_{m} N_{C}, \\
\frac{d V}{d t}= & F_{A}\left(N_{S} / V\right) N_{A} .
\end{aligned}
$$

Here, we introduced cell division and simulated the dynamics of only one daughter cell (to reduce the simulation time). When the cell volume $V$ reaches the division volume $V_{\text {div }}, V$ halves and chemicals are distributed to two daughter cells in equal probability. After computing these equations for a sufficiently long time under the $N_{S_{\text {ext }}^{\text {rich }}}$ condition, $N_{S_{\text {ext }}}$ suddenly changes to $N_{S_{\text {ext }}^{\text {poor }}}$ and is then set at this value over the starvation period $T_{\mathrm{stv}}$. Then, $N_{S_{\mathrm{ext}}}$ returns to the original value $N_{S_{\text {ext }}^{\text {irch }}}$. The lag time $\lambda$ is computed as the time needed to double the volume from $V_{0}$, i.e., the volume at which $S_{\text {ext }}$ recovers. The numerical results indicate that the absolute value of the correlation coefficient between $V_{0}$ and $\lambda$ is small. Here, the difference in $V_{0}$ in cells does not affect the distribution of the lag time. Stochastic simulation was carried out using the Gillespie algorithm. Parameter values were set to $V_{\text {div }}=2 \times 10^{3}$, $V_{\text {bath }}=1.0, N_{S_{\text {ext }}^{\text {rich }}}=10^{4}, N_{S_{\text {ext }}^{\text {poor }}}=10^{-3}$, and the others were the same as those described in Fig. 2. The length of starvation time $T_{\text {stv }}$ was set to $5 \times 10^{4}, 10^{6}, 2 \times 10^{6}$, and $10^{7}$ for Figs. 5(a) $-5(\mathrm{~d})$, respectively.

From the lag-time distribution obtained by numerical simulation, we can compute the peak and FWHM values directly. Since the experimental data do not include a sufficient amount of samples, we applied a smoothing filter to determine the FWHM, while the peak point was determined directly.

\section{APPENDIX D: ESTIMATED PARAMETER VALUES}

Here, we list the estimated value of parameters in Table II (for the detail of estimation procedure, see Sec. II. G).

TABLE II. Estimated parameter values.

\begin{tabular}{llcc}
\hline \hline Symbol & \multicolumn{1}{c}{ Meaning } & Estimated value (unit) & Reference \\
\hline$f_{0}$ & Stoichiometry of glucose and amino acids (measured by carbon, average) & $1.1(-)$ & {$[52]$} \\
$f_{1}$ & Stoichiometry of amino acids and average proteins & $209(-)$ & {$[53]$} \\
$f_{2}$ & Stoichiometry of amino acids and ribosomal protein & $7336(-)$ & {$[4]$} \\
$v$ & Synthesis rate of protein per ribosome $20(\mathrm{a} . \mathrm{a} / \mathrm{sec}) / 209(\mathrm{a} . \mathrm{a}) \times 3600(\mathrm{sec})$ & $345(1 / \mathrm{hour})$ & {$[4,53]$} \\
$m$ & Volume growth per synthesis of growth factor $1.0 \mu \mathrm{m}^{3}(E$. Coli volume) & $1.2 \times 10^{-2}(1 / \mathrm{mM})$ & {$[53]$} \\
& $\quad$ divided by $5 \times 10^{5}$ (\# of proteins) & $0.8(-)$ & {$[48]$} \\
$r$ & Fraction of actively translating ribosomes & $3.6 \times 10^{-3}(-)$ & Fitting \\
$\psi$ & Fraction of ribosomal proteins to component $A$ & $10^{5}(1 / \mathrm{mM} / \mathrm{hour})$ & Fitting \\
$D$ & Speed of phenomenological catabolism & $1.8 \times 10^{-2}(\mathrm{mM})$ & Fitting \\
$K$ & Saturation constant of $F_{A}+F_{B}$ & $2.3 \times 10^{-2}(\mathrm{mM})$ & Fitting \\
$K_{t}$ & Phenomenological constant changing $A / B$ balance & $10^{-3}(1 / \mathrm{mM} / \mathrm{hour})$ & Fitting \\
$k_{p}$ & Rate of association between $A$ and $B$ & $10^{-6}(1 / \mathrm{hour})$ & Fitting \\
$k_{m}$ & Rate of dissociation between $A$ and $B$ & &
\end{tabular}

[1] J. Monod, The Growth of Bacterial Cultures, Annu. Rev. Microbiol. 3, 371 (1949).

[2] S. J. Pirt, The Maintenance Energy of Bacteria in Growing Cultures, Proc. R. Soc. B 163, 224 (1965).

[3] M. Schaechter, O. Maaløe, and N.O. Kjeldgaard, Dependency on Medium and Temperature of Cell Size and Chemical Composition During Balanced
Growth of Salmonella Typhimurium, Microbiology 19, 592 (1958).

[4] M. Scott, C. W. Gunderson, E. M. Mateescu, Z. Zhang, and T. Hwa, Interdependence of Cell Growth and Gene Expression: Origins and Consequences, Science 330, 1099 (2010).

[5] P. M. Bennett and O. Maaløe, The Effects of Fusidic Acid on Growth, Ribosome Synthesis and RNA Metabolism in Escherichia Coli, J. Mol. Biol. 90, 541 (1974).

[6] N. Ishii, K. Nakahigashi, T. Baba, M. Robert, T. Soga, A. Kanai, T. Hirasawa, M. Naba, K. Hirai, A. Hoque et al., 
Multiple High-Throughput Analyses Monitor the Response of E. Coli to Perturbations, Science 316, 593 (2007).

[7] S. Klumpp, Z. Zhang, and T. Hwa, Growth Rate-Dependent Global Effects on Gene Expression in Bacteria, Cell 139, 1366 (2009).

[8] D. Madar, E. Dekel, A. Bren, A. Zimmer, Z. Porat, and U. Alon, Promoter Activity Dynamics in the Lag Phase of Escherichia Coli, BMC Syst. Biol. 7, 136 (2013).

[9] C. Furusawa and K. Kaneko, Zipf's Law in Gene Expression, Phys. Rev. Lett. 90, 088102 (2003).

[10] Y. Himeoka and K. Kaneko, Entropy Production of a Steady-Growth Cell with Catalytic Reactions, Phys. Rev. E 90, 042714 (2014).

[11] K. Kaneko, C. Furusawa, and T. Yomo, Universal Relationship in Gene-Expression Changes for Cells in SteadyGrowth State, Phys. Rev. X 5, 011014 (2015).

[12] J.-C. Augustin, L. Rosso, and V. Carlier, A Model Describing the Effect of Temperature History on Lag Time for Listeria Monocytogenes, International Journal of food microbiology 57, 169 (2000).

[13] O. Gefen, O. Fridman, I. Ronin, and N. Q. Balaban, Direct Observation of Single Stationary-Phase Bacteria Reveals a Surprisingly Long Period of Constant Protein Production Activity, Proc. Natl. Acad. Sci. U.S.A. 111, 556 (2014).

[14] F. K. Vasi and R. E. Lenski, Ecological Strategies and Fitness Tradeoffs in Escherichia Coli Mutants Adapted to Prolonged Starvation, Journal of genetics 78, 43 (1999).

[15] I. A. Swinnen, K. Bernaerts, E. J. Dens, A. H. Geeraerd, and J. F. Van Impe, Predictive modelling of the microbial lag phase: a review. International journal of food microbiology, Int. J. Food Microbiol. 94, 137 (2004).

[16] C. Prats, D. López, A. Giró, J. Ferrer, and J. Valls, Individual-Based Modelling of Bacterial Cultures to Study the Microscopic Causes of the Lag Phase, J. Theor. Biol. 241, 939 (2006).

[17] J. Baranyi, P. J. McClure, J. P. Sutherland, and T. A. Roberts, Modeling Bacterial Growth Responses, Journal of industrial microbiology 12, 190 (1993).

[18] I. Levin-Reisman, O. Gefen, O. Fridman, I. Ronin, D. Shwa, H. Sheftel, and N.Q. Balaban, Automated Imaging with ScanLag Reveals Previously Undetectable Bacterial Growth Phenotypes, Nat. Methods 7, 737 (2010).

[19] A. Jõers and T. Tenson, Growth Resumption from Stationary Phase Reveals Memory in Escherichia Coliz Cultures, Sci. Rep. 6, 24055 (2016).

[20] A. Maitra and K. A. Dill, Bacterial Growth Laws Reflect the Evolutionary Importance of Energy Efficiency, Proc. Natl. Acad. Sci. U.S.A. 112, 406 (2015).

[21] S. A. Kauffman, The Origins of Order: Self-Organization and Selection in Evolution (Oxford University, New York, 1993).

[22] D. Segré, D. Ben-Eli, and D. Lancet, Compositional Genomes: Prebiotic Information Transfer in Mutually Catalytic Noncovalent Assemblies, Proc. Natl. Acad. Sci. U.S.A. 97, 4112 (2000).

[23] K. Kaneko, Recursiveness, Switching, and Fluctuations in a Replicating Catalytic Network, Phys. Rev. E 68, 031909 (2003).

[24] Y. Himeoka and K. Kaneko, Enzyme Oscillation Can Enhance the Thermodynamic Efficiency of Cellular
Metabolism: Consequence of Anti-phase Coupling Between Reaction Flux and Affinity, Phys. Biol. 13, 026002 (2016).

[25] The model equation (2) is nondimensionalized by appropriate normalization.

[26] F. C. Nucifora, M. Sasaki, M. F. Peters, H. Huang, J. K. Cooper, M. Yamada, H. Takahashi, S. Tsuji, J. Troncoso, V. L. Dawson et al., Interference by Huntingtin and Atrophin-1 with CBP-Mediated Transcription Leading to Cellular Toxicity, Science 291, 2423 (2001).

[27] Y. Chai, J. Shao, V. M. Miller, A. Williams, and H. L. Paulson, Live-Cell Imaging Reveals Divergent Intracellular Dynamics of Polyglutamine Disease Proteins and Supports a Sequestration Model of Pathogenesis, Proc. Natl. Acad. Sci. U.S.A. 99, 9310 (2002).

[28] R. S. Rajan, M. E. Illing, N. F. Bence, and R. R. Kopito, Specificity in Intracellular Protein Aggregation and Inclusion Body Formation, Proc. Natl. Acad. Sci. U.S.A. 98, 13060 (2001).

[29] Y. Maki, H. Yoshida, and A. Wada, Two Proteins, YfiA and YhbH, Associated with Resting Ribosomes in Stationary Phase Escherichia Coli, Genes Cells 5, 965 (2000).

[30] M. Ueta, R. L. Ohniwa, H. Yoshida, Y. Maki, C. Wada, and A. Wada, Role of HPF (Hibernation Promoting Factor) in Translational Activity in Escherichia Coli, J. Biochem. (Tokyo) 143, 425 (2008).

[31] A. Vila-Sanjurjo, B. S. Schuwirth, C. W. Hau, and J. H. D. Cate, Structural Basis for the Control of Translation Initiation During Stress, Nat. Struct. Mol. Biol. 11, 1054 (2004).

[32] J. Tyedmers, A. Mogk, and B. Bukau, Cellular Strategies for Controlling Protein Aggregation, Nat. Rev. Mol. Cell Biol. 11, 777 (2010).

[33] J. J. Hopfield, Kinetic Proofreading: A New Mechanism for Reducing Errors in Biosynthetic Processes Requiring High Specificity, Proc. Natl. Acad. Sci. U.S.A. 71, 4135 (1974).

[34] See Supplemental Material at http://link.aps.org/ supplemental/10.1103/PhysRevX.7.021049 for analytic calculation of lag time, detailed conditions to obtain main results presented in main article, and two additional figures.

[35] R. Hengge-Aronis, Regulation of Gene Expression During Entry into Stationary Phase, in Escherichia Coli and Salmonella Typhimurium edited by F. C. Neidhardt (ASM Press, Washington, 1996), pp. 1497-1512.

[36] H. E. Schellhorn, J. P. Audia, L. I. C. Wei, and L. Chang, Identification of Conserved, rpoS-Dependent StationaryPhase Genes of Escherichia Coli, J. Bacteriol. 180, 6283 (1998).

[37] W. J. Penfold, On the Nature of Bacterial Lag, J Hygiene 14, 215 (1914).

[38] S. John Pirt et al., Principles of Microbe and Cell Cultivation (Blackwell Scientific Publications, Hoboken, New Jersey, 1975), p. 11.

[39] T.E. Oscar, Validation of Lag Time and Growth Rate Models for Salmonella Typhimurium: Acceptable Prediction Zone Method, J. Food. Sci. 70, M129 (2005).

[40] C. Pin and J. Baranyi, Single-Cell and Population Lag Times as a Function of Cell Age, Appl. Environ. Microbiol. 74, 2534 (2008).

[41] Here, $T_{\text {stv }}$ is computed from the point in time at which the external substrate concentration starts to decrease 
(i.e., $T_{\text {dec }} \leq T_{\text {stv }}$ ); if it was computed from the time when the depletion was computed, a slower decrease with the increase of $T_{\text {dec }}$ would effectively elongate the starvation time itself.

[42] S. H. Strogatz, Nonlinear Dynamics and Chaos: With Applications to Physics, Biology, Chemistry, and Engineering (Westview Press, Boulder, Colorado, 2014).

[43] D. T. Gillespie, Exact Stochastic Simulation of Coupled Chemical Reactions, J. Phys. Chem. 81, 2340 (1977).

[44] Note that the log-normal or Gamma distribution of chemicals in a cell has been well observed and mathematically explained for an exponentially growing cell $[45,46]$.

[45] C. Furusawa, T. Suzuki, A. Kashiwagi, T. Yomo, and K. Kaneko, Ubiquity of Log-Normal Distributions in Intracellular Reaction Dynamics, Biophysik 1, 25 (2005).

[46] L. Cai, N. Friedman, and X. S. Xie, Stochastic Protein Expression in Individual Cells at the Single Molecule Level, Nature (London) 440, 358 (2006).

[47] A. Elfwing, Y. LeMarc, J. Baranyi, and A. Ballagi, Observing Growth and Division of Large Numbers of Individual Bacteria by Image Analysis, Appl. Environ. Microbiol. 70, 675 (2004).

[48] H. Bremer and P. P. Dennis, Modulation of Chemical Composition and Other Parameters of the Cell at Different Exponential Growth Rates, EcoSal. Plus 3 (2008).

[49] J. Forchhammer and L. Lindahl, Growth Rate of Polypeptide Chains as a Function of the Cell Growth Rate in a Mutant of Escherichia Coli 15, J. Mol. Biol. 55, 563 (1971).

[50] Recall that Eq. (2) is already nondimensionalized with appropriate scaling. To make a quantitative comparison with experimental data, we replaced each term in Eq. (2) as follows: $\quad S_{\text {ext }} \rightarrow m S_{\text {ext }} / f_{1}, \quad S \rightarrow m S / f_{1}, \quad A \rightarrow m A$, $B \rightarrow m B, C \rightarrow m C, v \rightarrow v \psi r /\left(f_{0} D / m\right), k_{p} \rightarrow k_{p} /\left(f_{0} D\right)$, $k_{m} \rightarrow k_{m} /\left(f_{0} D / m\right), \quad K \rightarrow m K / f_{1}, \quad K_{t} \rightarrow m K_{t} / f_{1}$, and $t \rightarrow t\left(f_{0} D / m\right)$, with additional parameters $f_{0}, f_{1}, r, m$, $\psi$, and $D$, which indicate the stoichiometry between external substrates and substrates, stoichiometry between substrates and macromolecular components (components $A$ and $B$ ), fraction of ribosomal proteins to component $A$, fraction of actively translating ribosomes, volume growth per synthesis of component $\mathrm{A}$, and speed of the $S_{\text {ext }} \leftrightarrow S$ reaction, respectively. To fit the experimental data, we adopt the interpretation that the external substrate, substrate, and both components A and B correspond to glucose, amino acids, and proteins, respectively. Thus, we adopt the stoichiometry between glucose and amino acids, that between amino acids and typical (average size of) proteins, and that between amino acids and ribosomal proteins as $f_{0}, f_{1}$, and $f_{2}$, respectively. To compare with the data of Refs. [4,45,46], the ribosomal fraction $\phi$ is defined as $\phi=$ $\psi\left(f_{2} / f_{1}\right)[(A+C) /(A+B+2 C)]$. (In the denominator, $2 C$ is introduced because the relationship concerns the molecular weight rather than the number, and complex $\mathrm{C}$ consists of $\mathrm{A}$ and B.).

[51] The addition of antibiotics may correspond to the increase in $k_{p}$ in our model, while Scott et al. [4] showed experimentally that the addition of antibiotics increases the $y$ offset of the linear relation.

[52] ChemSpider, http://www.chemspider.com.

[53] R. Milo, R. Phillips, and O. Nigel, Cell Biology by the Numbers (Garland Science, New York, 2016).

[54] M. K. Sands and R. B. Roberts, The Effects of a TryptophanHistidine Deficiency in a Mutant of Escherichia Coli, J. Bacteriol. 63, 505 (1952).

[55] G.S. Stent and S. Brenner, A Genetic Locus for the Regulation of Ribonucleic Acid Synthesis, Proc. Natl. Acad. Sci. U.S.A. 47, 2005 (1961).

[56] B. J. Paul, M. M. Barker, W. Ross, D. A. Schneider, C. Webb, J. W. Foster, and R. L. Gourse, DKSA: A Critical Component of the Transcription Initiation Machinery that Potentiates the Regulation of rRNA Promoters by ppGpp and the Initiating NTP, Cell 118, 311 (2004).

[57] M. Kajitani and A. Ishihama, Promoter Selectivity of Escherichia Coli RNA Polymerase. Differential Stringent Control of the Multiple Promoters from Ribosomal RNA and Protein Operons, J. Biol. Chem. 259, 1951 (1984).

[58] A. Raghavan and D. Chatterji, Guanosine TetraphosphateInduced Dissociation of Open Complexes at the Escherichia Coli Ribosomal Protein Promoters rplJ and rpsA Pl: Nanosecond Depolarization Spectroscopic Studies, Biophys. Chem. 75, 21 (1998).

[59] M. M. Barker, T. Gaal, C. A. Josaitis, and R. L. Gourse, Mechanism of Regulation of Transcription Initiation by ppGpp. I. Effects of ppGpp on Transcription Initiation in vivo and in vitro, J. Mol. Biol. 305, 673 (2001).

[60] L. U. Magnusson, A. Farewell, and T. Nyström, ppGpp: A Global Regulator in Escherichia Coli, Trends Microbiol. 13, 236 (2005).

[61] L. U. Magnusson, T. Nyström, and A. Farewell, Underproduction of $\varsigma^{70}$ Mimics a Stringent Response a Proteome Approach, J. Biol. Chem. 278, 968 (2003). 